\title{
A Study on the Ethical and Moral Responsibility Problem Related to Self-driving System
}

\author{
Zion Hwang1)
}

\begin{abstract}
As AI technology develops, research on developing self-driving cars is being carried out actively. Self-driving cars can reduce traffic accidents and bring convenience as a means of transportation. For self-driving cars to be commercialized, detailed standards of judgment must be prepared for emergency crisis response while driving. In response, this paper examines ethical/moral issues regarding the operation of self-driving cars. A prior study on ethical and moral issues related to self-driving cars found that there were different standards for judging emergency situations, depending on country, age, occupation and gender. This paper suggests that disputes such as human rights issues, privacy issues, and the possession of responsibility for traffic accidents must be resolved before autonomous driving can be implemented, and that detailed and clear legal rules must be followed.
\end{abstract}

Keywords : $4^{\text {th }}$ Industrial Revolution, Self-driving Cars, Ethical Decision-making, Moral Decision-making, Trolley dilemma

\section{Introduction}

Cars greatly increased the mobility of people and goods, which served as the basis for development throughout the industry. Car accidents are one of the major causes of death for people, although there are many advantages caused by cars. As a result, studies related to driver safety have been conducted continuously, and with the advent of AI technology, studies on self-driving cars for driver safety have been actively carried out[1]. People expect that commercialization of self-driving cars will completely reduce traffic accidents, but it will be hard to completely eliminate them. Even during self-driving, sudden emergencies on the road as they are now will always occur, and by the judgment of the vehicle, either the occupants or the pedestrians can be victims of traffic accidents.

Received(May 1, 2019), Review Result(1st: May 27, 2019, 2nd: July 31, 2019), Accepted(September 10, 2019)

1) (Assistant Professor) 18330 Department of Smart Systems Software Engineering, Hyupsung Univ., 72 Choerubaek-Ro, Bongdam-Eup, Hwaseong-Si, Gyeonggi-Do, Korea

email: zhwang@uhs.ac.kr 
This study focuses on ethical and moral responsibility issues and examines them in depth in relation to self-driving and traffic accidents. To help understand self-driving cars, we will look at the key technologies and the types and characteristics of self-driving cars. It then introduces the results of 'Morality Machine Study' conducted by MTT that plays a pioneering role in the ethical problems of self-driving cars, and then presents implications for the ethical and moral aspects that need to be addressed in more depth in the future.

\section{Theoretical Background}

\subsection{Self-driving Car Core Technology}

Self-driving cars are equipped with technology that enables a car to recognize its driving environment and move it to its destination without being operated by a person. Various technologies have been developed so far for self-driving cars, and among them, the representative technologies currently being applied are as follows.

Key technologies include Highway Driving Assist (HDA), a technology that automatically maintains distance between cars, a technology that detects and alarms surrounding vehicles during reversing, and a technology called Reverse Alert (BSD) and Lane Departure Warning War System (LDWS). Other key technologies are the 'Automatic Emergency Braking System (AEB), which operates the braking system if the vehicle is not recognized while driving, the 'LKAS' (Lane Keeping Assist System), a technology that complements situations outside the lane of driving without directional instructions, and the 'Smart Assist System' which maintains the distance between the cars at the set speed.

\subsection{Self-driving stage}

The Society of Automotive Engineers (SAE) divides self-driving cars into six stages, in [Table 1] from Level 0 to Level 5 as follows[2].

[Table 1] Self-driving cars into six stages[2]

\begin{tabular}{|l|l|}
\hline \multicolumn{1}{|c|}{ Levels } & \multicolumn{1}{c|}{ Contents } \\
\hline Level 0 (non-automatic) & Step without autonomous driving \\
\hline Level 1 (driver assisted) & Step to control some speed and braking \\
\hline Level 2 (partial automation) & The step of self-controlled speed and direction \\
\hline Level 3 (conditional automation) & $\begin{array}{l}\text { A step that reduces driver intervention and recognizes traffic } \\
\text { signals and road traffic }\end{array}$ \\
\hline
\end{tabular}




\begin{tabular}{|l|l|}
\hline Level 4 (altitude automation) & The stage where the driver only needs to set the destination \\
\hline Level 5 (fully automated) & Close to unmanned vehicles \\
\hline
\end{tabular}

Level 0 is a stage where there is no technology related to autonomous driving, and it is the stage where the driver directly controls all operations required for driving the vehicle. Level 1 is a stage where autonomous driving technology is used a little, and it selects and controls speed and direction by using camera and sensor. Cruise control, lane departure alarms and crash warning systems. Level 2 is a step closer to the appearance of an autonomous vehicle, and the vehicle controls its own speed and direction. Level 3 is a step where driver intervention is gradually reduced, and the vehicle can detect and avoid obstacles on its own. Even in the autonomous mode, it is possible to secure the safety through the function of requesting the driver's intervention according to the situation. Level 4 is the stage where fully autonomous driving is possible, which is mainly mentioned in the automobile industry, and autonomous driving car is at this stage. Car monitors the whole moving section, and car judges and carries out safety functions by itself. However, the driver only needs to input the route to the destination before departure. Level 5 is the stage where full automation is realized and can be expressed in general as an unmanned vehicle. Since the concept of the occupant is stronger than the concept of the driver and there is virtually no intervention of the person (driver), the inside of the vehicle will be changed into a design that enhances the comfort of the occupant because there are no controllable accessories (steering wheel, brake pedal, driver's seat, etc.)

Level 2 vehicles are already being commercialized in many OEMs, and in Korea, including Cadillac and Audi, Hyundai Motor is also currently carrying out a self-driving test run on roads that are now allowed to autonomously drive in 2020 with the aim of mass-production level 3 vehicles. With Level 3, it can be excluded to give a warning if the driver takes off the steering wheel for more than a certain amount of time, such as in a recently mass-produced vehicle. In other words, the vehicle directly controls the steering wheel, brakes and accelerator to maintain the vehicle-to-vehicle distance and to drive, accelerate and stop on major roads. Technologically, rapid progress is being made. In the case of a recent self-driving car accident, the accident occurred due to an exception situation where it was difficult to handle when the driver had no forward-looking or control due to the safety of the vehicle's operation.

Level 2 vehicles are already being commercialized by many OEMs, and Hyundai Motors, including Cadillac and Audi, are also conducting self-propulsion test runs on roads that are allowed to autonomously drive in 2020 with the aim of mass production of level 3 vehicles. 
With Level 3, it can be excluded to give a warning if the driver takes off the steering wheel for more than a certain amount of time, such as in a recently mass-produced vehicle. That is, the vehicle can directly control the steering wheel, the brake, and the accelerator on the main road, thereby maintaining the inter-vehicle distance and performing the operation, acceleration, and stopping. This technological progress is making drastic progress. In the case of a recent accident involving an autonomous vehicle accident, an accident occurred due to an exceptional situation which is difficult to handle in a situation in which the driver does not have the front view and control right due to safety of the vehicle. We expect to be able to reach level 5 by 2025 even in this exceptional situation. Even with the technical foundation in place, controversy over the judgment standard is still intensifying.

In order to move into the era of Level 5, people's value judgment data needs to be collected and analyzed to program how self-driving vehicles should be judged and determined in the event of countless crises. In addition, since autonomous driving can cause driver interference at levels 2 to 4 , the next chapter will look at people's perceptions about the standards of self-driving vehicles related to coping with crises, and will look at problems based on them, and discuss future issues.

\section{Analysis of Ethical Consciousness in Autonomous Driving}

\subsection{Priority in Autonomous Driving Environment}

When a vehicle encounters an unexpected emergency during self-driving, it will follow programmed standards to minimize human and material damage. A number of items will need to be considered when setting the standard. However, the idea of item selection and item importance will not be consistent with everyone. In this regard, MIT's Media $\mathrm{Lab}$ is collecting people's data on the selection of these items and their importance by item through the 'Moral Machine' website[3]. The main analysis of research results conducted to date is as follows.

In 18 months, 233 countries and 2,300,000 people participated in the case analysis of ethical judgment in 4,000 autonomous driving situations. According to the results, the ethical standards have shown a greater importance to people than pets, to the majority, to women rather than men, to athletes rather than obese, to business executives rather than homeless or criminals.

In many countries, we are working to define a system of unified ethical rules. However, according to the analysis, it was clear that the conscious differences among countries were 
clear. In [Figure 1], the closer to 1 , the younger, the closer to -1 , the older. As shown in [Figure 1] and [Figure 2], countries with strong individualism tend to view life and safety of young people as important[1][4][5]. In [Figure 2], the closer to 1 means more people, the closer to -1 , the more personal it means[1][4][5]. However, these European and North American countries have a number of life preferences rather than individuals. In Britain and the United states, we can confirm that more people's lives and safety have been judged by more important standard than by all other choices. However, in most Asian countries, it is a priority for the elderly, but it can be seen that the life of the individual is more important than the majority.

The basic concept of existing automobiles was to minimize the exposure to risks through various devices for driver safety[6]. Therefore, the car is made entirely considering the safety of the driver, and the judgment in the risk situation for the driver and the pedestrian was the responsibility of the driver. However, in the autonomous driving situation, this judgment should be performed by the vehicle first. In Moral Machine identification example, we make a selection if there is a pedestrian in the avoiding position in situations where sudden obstacle or risk factors require avoidance action. It is also possible to determine the number of pedestrians and the number of passengers in the vehicle at this time. Under these circumstances, giving priority to the majority should consider whether it is appropriate to save the majority even if an accident kills minority. It also matters whether it is appropriate to expose pedestrians to danger to save passengers in the vehicle.

During current car accidents, many accidents are caused by driver inability to judge due to this critical situation. In such a case, the driver may be able to avoid ethical responsibility of the accident due to the inexperienced driving or confusion of judgment. However, in self-driving vehicles, it is difficult to avoid ethics because in such a situation, the automatic operation is made through ethical decision-making. The driver may be removed from ethical responsibility at this time.

Nevertheless, the ethical decision-making itself is a consensus-based judgment, which will be linked to the problem of social and personal consciousness. Technological maturity for self-driving is rapidly improving and already has the ability to drive with a lower accident rate than people on significant roads. But if the agreement allows drivers to be exposed to danger at any time based on the judgment of the vehicle, we have to think about whether it will be possible for drivers to shift their gaze away from driving through self-driving. And if the driver can not turn his gaze to another, there is no doubt about the need for autonomous driving. Of course, the likelihood of an accident will be significantly lower than it is now, but 
even if that includes any ethical judgment that the driver may be at risk, he or she will endeavor to make it possible for anyone to live in that situation. So the choice in the majority and in the individual should be considered, including awareness of essential driving[7][8]. And if the driver can't turn his eyes away, naturally doubts arise about the need for autonomous driving. Of course, the likelihood of an accident will be significantly lower than it is today, but if that includes ethical judgments that a driver can be dangerous, it would be an effort to ensure that anyone has the potential to live in that situation. Therefore, the choice of the majority and the individual in a sudden accident should be considered fundamentally, including a sense of driving.

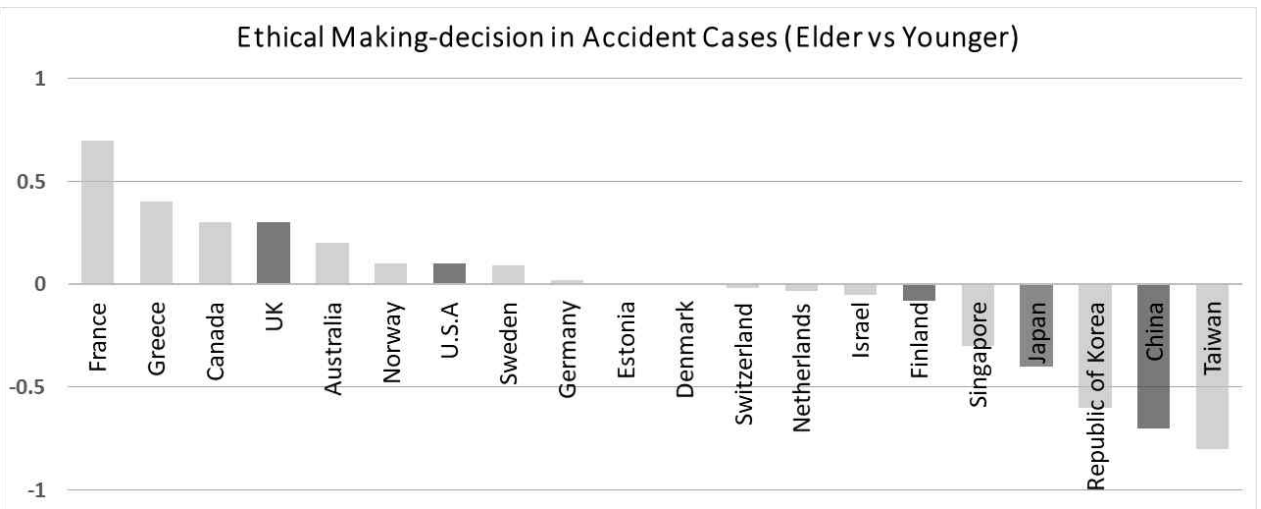

[Fig. 1] Ethical Making-decision in Accident Cases (Elder vs Younger)[1][4][5]

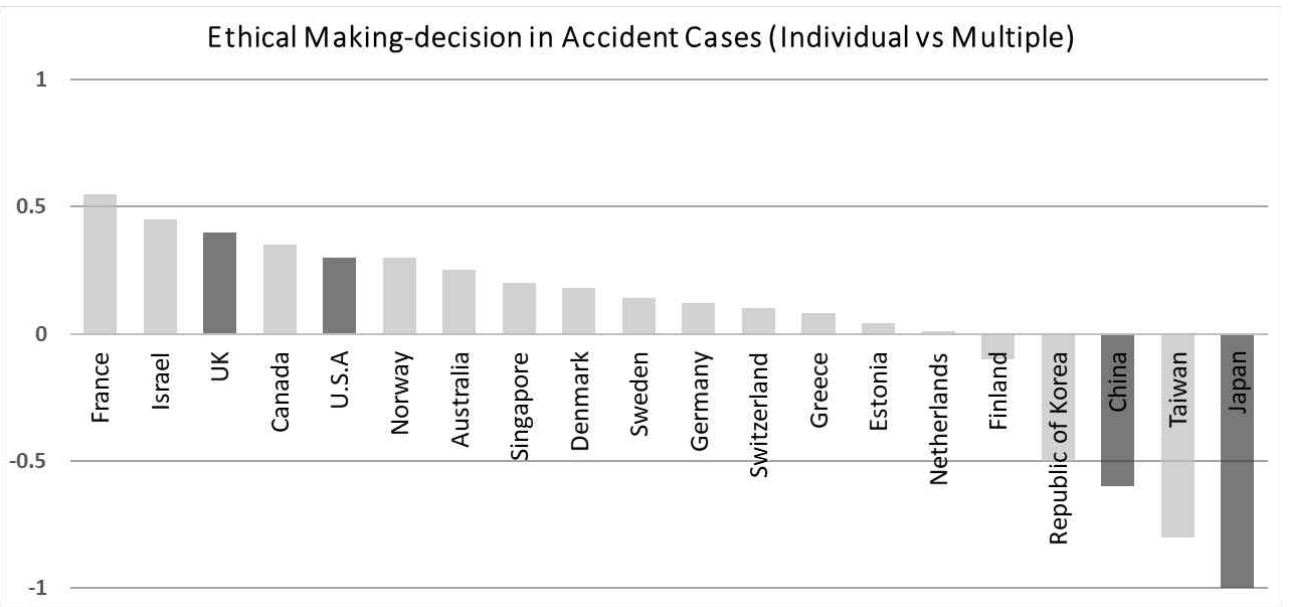

[Fig. 2] Ethical Making-decision in Accident Cases (Individual vs Multiple)[1][4][5] 
The richer the country regards the life of the unauthorized crosser as less important than the people of the poorer countries, and the more the gap between the rich and the poor, the more important the safety of the person with social status than the homeless. It can be seen that the characteristics between countries are quite different, but the geographically neighboring countries have a similar pattern, which can be seen as South America, with Europe and North America including Western, Oriental, Central and South America and Oceania[9]. However, Japan is the country that places the most importance on pedestrians, while China is also the country where the passengers are given top priority.

Although patents and standards have been laid out in response to countermeasures against risk of autonomous driving, it is difficult to establish consistent global ethical standards in different situations, such as countries and regions. The accidents of existing vehicles were taken into consideration for the safety of the driver and the judgment of the external situation was entirely for the driver. In situations where it is difficult for the driver to judge, the driver must take responsibility for the result, which will be finalized according to the legal regulations of the country concerned. However, the autonomous driving situation requires that the driver and the external situation are determined according to the vehicle, that is, the already defined ethical rule.

The point to note here is that you can not define all the situations in advance, so you have to specify the priority for people like a moral machine. And these priorities should include values that can be agreed by the majority of people in the world, region, or country.

\subsection{Ethical and moral considerations to be considered in the future}

In the autonomous driving situation as described above, the subject of judgment becomes the system of the vehicle, that is, programmed ethical and moral rule. However, the standards for self-driving cars can lead to very serious ethical and moral problems related to human rights. There is also a need for a criterion to determine the dilemma caused by the driver's interference with the autonomous driving and the unethical demands of the driver. Autonomous driving examines the possibility of various evasive actions against the accident situation, finds a countermeasure, and applies ethical judgment in situations where it is considered difficult to evade. However, if there is a possibility of avoidance, and the driver intervenes at the time of control, it should be able to clarify responsibility judgment and grounds when an accident occurs while the 
response is delayed and the above judgment process is in progress.

Even if autonomous vehicles are deployed, there will be a long period of time when the transitional period, that is, the autonomous driving vehicles and the vehicles that are not subjected to semi-autonomous driving or autonomous driving are mixed. In this case, more complex ethical debates arise.

The above-mentioned problems can be summarized as follows. First, the issue of inequality related to human rights. Prioritizing a person's gender, age, occupation, or social class will result in differentiating human rights. In the end, there is a risk of becoming an anti-human rights society that leads to a return to the hierarchical society of the past. Second, the issue of privacy Self-driving cars will determine and respond to priorities based on personal data from drivers, passengers and passers-by in times of crisis. It should be checked closely to ensure that the privacy laws are maintained and that personal information needed for autonomous driving can be provided. It is questionable how much information a self-driving car will make perfect judgment of a crisis. Third, the issue of identifying the cause of the accident Level 2 Level 4 allows driver intervention, which may result in driver-induced traffic accidents. Regardless of driver intervention, however, a traffic accident may occur due to program errors or technical defects in the vehicle. In the event of an accident due to a problem with a driver or self-driving car, the standard of responsibility needs to be clearly set.

\section{Conclusion}

This paper discussed ethical/moral issues that were judged must be considered before the commercialization of self-driving cars.

Before the commercialization of self-driving cars, in-depth discussions and agreements should be made on gender, age, occupation and differences among countries on ethical/moral standards. This is because the standards proposed to solve ethical/moral problems can in effect lead to class inequality, excessive disclosure of personal information. In addition, when a crisis situation occurs due to human intervention during autonomous driving, the standard for determining the responsibility for a traffic accident shall be prepared.

Self-driving cars will provide convenience to users. However, even if various technological advances are made regarding self-driving cars, actual autonomous driving such as Level 5 will be possible only when human rights, ethical and moral issues are agreed upon at the same time. As can be seen from the results of the 'Moral Research' 
there are various perspectives on each criterion, so the relevant studies should be more active and studies using qualitative data as well as quantitative data should be conducted.

Therefore, the commercialization of self-driving cars will be completed only when the efforts of manufacturing companies, insurance companies, countries support to draw up legal systems and researchers for self-driving cars come together.

\section{References}

[1] https://www.technologyreview.com/ Mar 19 (2018)

[2] https://blog.naver.com/mage7th/221499410042, Oct 3 (2018)

[3] http://moralmachine.mit.edu/, Jun 24 (2016)

[4] Karen Hao, Should a Self-Driving Car Kill the Baby or the Grandma? Demands on Where you're from?, MIT Technology Review, (2018)

[5] https://www.technologyreview.com/s/612341/a-global-ethics-study-aims-to-help-ai-solve-the-self-driving-trolley -problem/, Oct 24 (2018)

[6] Il Han Bae, Kwang Hyung Lee, Criteria of the Prior Protection in Fully Autonomous Car Accidents, Korean Journal of Futures Studies, (2018), Vol.3, No.1, pp.25-48.

[7] Ethically Aligned Design-Version II, IEEE, (2017)

[8] Self-Driving Cars and Insurance, Insurance Information Institute, (2016)

[9] Pillath Susanne, Automated Vehicles in the EU, European Parliamentary research Service, (2016) 